\title{
Factor XI Defieiency: A Rare Bause of Prolonged Bleeding in a Post-Operative Patient
}

\section{Sumit Barik', Sweta ${ }^{2}$}

From the Department of Pathology and Hematology', Department of Transfusion

Medicine and Blood Banking ${ }^{2}$; ESI Post graduate Institute of Medical Sciences and

Research, New Delhi-110015, India.

\section{Abstract:}

A boy aged 14 years was operated for a cyst in oral cavity. The post-operative stay was complicated by continued bleeding from the site of incision in oral cavity. The bleeding time was found to be normal however coagulation time was found to be 25 minutes. History of any abnormal bleeding in past was not available, parents refused to have noted any swelling in joints. Further evaluation showed abnormally prolonged APTT however PT was normal. Based on mixing experiments and factors assays, diagnosis of factor XI deficiency was made.

Key words: Cysts, Factor XI deficiency, Bleeding Time, Hemorrhagic disorders, Hemorrhage.

\section{Introduction}

Factor XI deficiency is a rare disorder which is also known as hemophilia $C$. It was noted first time in the year 1953 [1]. The deficiency of factor XI usually manifests as delay in haemostasis after surgical procedures or trauma though severe deficiency of factor XI (factor levels <15\%) may present as bleeding tendency. The coagulation tests show prolonged activated partial thromboplastin time (APTT). Hemophilia A and B are usually thought as first diagnosis specially in males whenever a prolonged APTT is noted, though deficiency of factor XI may cause similar illness. The low incidence and rarity of this deficiency producing significant complication compelled us to report this case.
Case Report

A 14 year old boy was brought in surgical OPD by his parents for a cyst in oral cavity which was noted about 30 days back by the patient. The clinical examination showed cyst was about $1.5 \mathrm{~cm}$ in diameter. General physical and systemic examination did not show any other abnormality. Minor surgery under local anaesthesia was advised for cyst removal and biopsy. The post-operative stay was complicated by prolonged bleeding. The boy was sent to Hematology Department for further evaluation to rule out hemophilia. A detailed history was taken from the patient and parents. The parents never noticed prolonged bleeding when the boy sustained injury while playing. The

Corresponding Author: Dr. Sweta

Email: sweta20eleven@gmail.com

Received: March 12, 2014 | Accepted: April 30, 2014 | Published Online: May 25, 2014

This is an Open Access article distributed under the terms of the Creative Commons Attribution License (creativecommons.org/licenses/by/3.0)

Conflict of interest: None declared | Source of funding: Nil | DOl: http://dx.doi.org/10.17659/01.2014.0043 
boy had occasional epistaxis in the past but never a prolonged one. There was no history of easy bruising, bleeding through mouth and hematuria. Patient was not taking any drug for any chronic illness.

Hematological investigations included platelet counts, bleeding time, coagulation time, PT and APTT. Clotting time was increased which showed coagulation factor deficiency. PT was found within normal limits. APTT was found to be 90 seconds compared to control of 35 seconds. A normal PT ruled out deficiency of factors I, II, V, VII, and X. Standalone prolonged APTT signified a deficiency or inhibition of one or more of factors VIII, IX, XI and factor XII [Table 1].

Mixing experiments were done with fresh plasma, adsorbed plasma and aged plasma. The fresh plasma corrected the abnormal APTT. Correction done by fresh plasma ruled out inhibitors of any factor. The adsorbed plasma is deficient in factor II, VII, IX, and X and correction of APTT rules out deficiency of these factors and specially IX in this patient. Aged plasma is deficient in factor $\mathrm{V}, \mathrm{VIII}$ and APTT correction rules out deficiency of these factors and specially factor VIII in this patient. Activity of factor VIII and IX were found normal. The deficiency of Hageman factor or factor XII was not considered reason for bleeding as this never produces a prolonged bleeding in patients and only detected accidentally in laboratory tests. The diagnosis of factor XI deficiency was made on basis of these results [Table 2].

The levels of factor XI showed moderate deficiency. The level of activity of factor XI and plasma levels are shown in Table 3. The patient was treated with fresh plasma and haemostasis was achieved.

\section{Discussion}

Our patient was diagnosed as hemophilia C. Incidence of Hemophilia C is 1 in $10,00,000$ in general population. A higher incidence is seen in Jews [2]. The disease is inherited as incomplete

Table 1: Results of haematological tests and coagulation profile

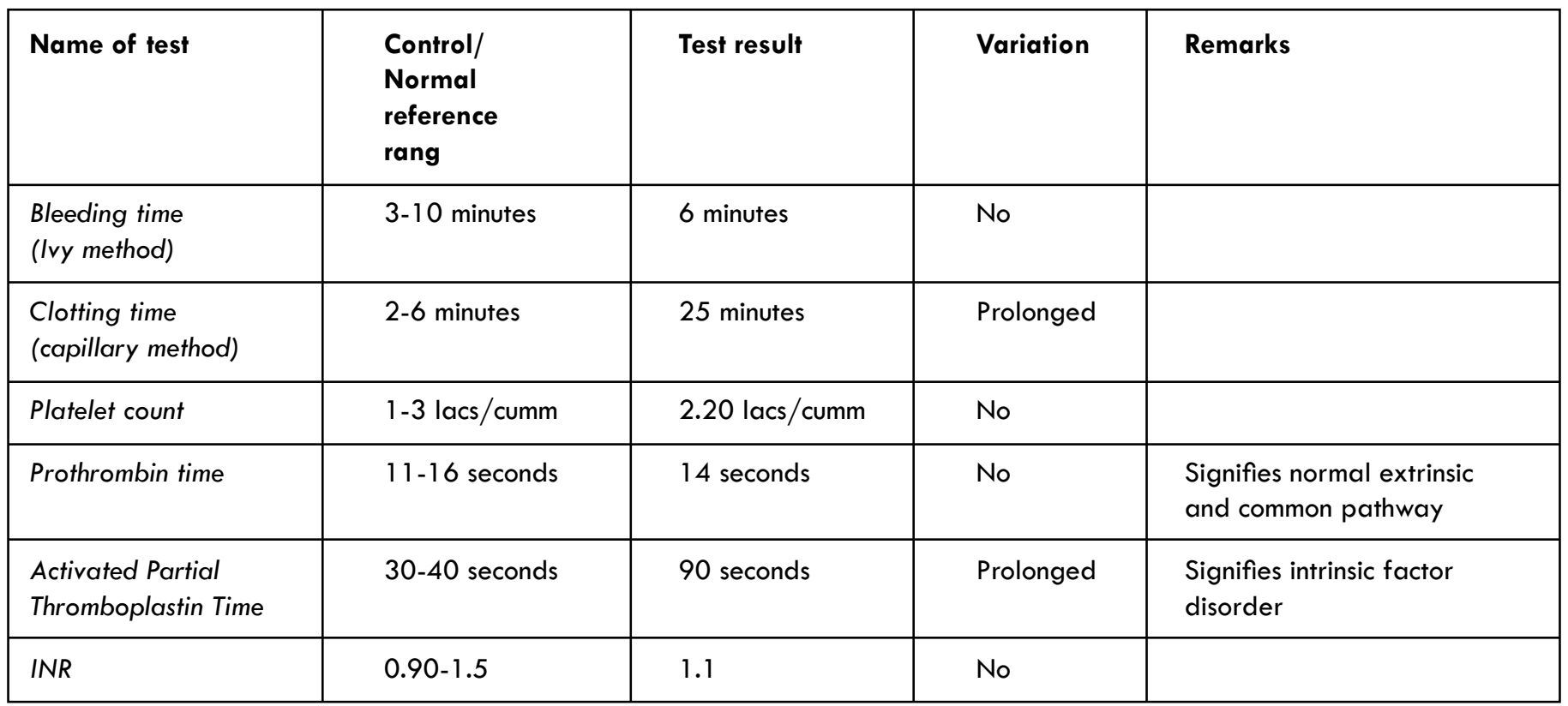

PT: Prothrombin Time; APTT: Activated Partial Thromboplastin Time; INR: International Normalized Ratio 
autosomal recessive pattern. The mutation as high as $5 \%$ to $11 \%$ in Ashkenazi Jews has been reported [3]. Gene for factor eleven is located on chromosome 4 long arm band 3 . The deficiency is usually manifested after surgical trauma or injuries however severe factor XI deficiency can produce bleeding tendency. The usual presentation of epistaxis, hematuria and menorrhagia which are found in other coagulation disorders can also be found in factor XI deficiency though haemarthrosis is rare.

Our patient had a surgical procedure in oral cavity which manifested as prolonged bleeding. He never had any bleeding tendency or haemarthrosis in past. The factor XI deficiency in this patient manifested after minor surgery in oral cavity which has higher fibrinolytic activity. Higher incidence of hemophilia $A$ and $B$ in general population and male sex were considered in this patient and coagulation studies were asked. The APTT confirmed intrinsic factor deficiency. Normal factor VIII and IX activity and absence of inhibitors lead us to think of factor XI deficiency. Factor XII which is another factor in intrinsic pathway never causes a prolonged bleeding in patients. The factor XI deficiency was moderate and activity was $20 \%$. Moderate deficiency of factor XI do not cause tendency to bleed and only manifests after surgical procedures or trauma. Splicing, point mutation with substitution of amino acid and a stop codon are types of mutation seen in this deficiency. Splicing is seen in general population [4]. Various studies have confirmed that bleeding can occur in heterozygotes [5-7]. This disorder is, therefore, not completely recessive and the variability of the bleeding tendency constitute one enigma of this deficiency.

Our patient was treated with fresh plasma and haemostasis was achieved. The deficiency is treated and prevented by using fresh plasma, plasma concentrates and recombinant factor XI.
Table 2: APTT correction of patient's sample in mixing experiments

\begin{tabular}{|ll|}
\hline Plasma & Interpretation \\
\hline Adsorbed plasma & Corrected \\
\hline Fresh plasma & Corrected \\
\hline Aged plasma & Corrected \\
\hline Factor VIII deficient plasma & Corrected \\
\hline Factor IX deficient plasma & Corrected \\
\hline
\end{tabular}

Table 3: Factor XI levels in patient

\begin{tabular}{|l|l|l|}
\hline Factor & Levels & Reference Range \\
\hline Factor XI activity & $20 \%$ & $70-120 \%$ \\
\hline Factor XI levels & $35 \mathrm{IU} / \mathrm{dL}$ & $65-120 \mathrm{IU} / \mathrm{dL}$ \\
\hline
\end{tabular}

\section{Conclusion}

Factor XI deficiency remains challenging because the factors influencing bleeding risk are still not fully understood and heterozygous patients also can show prolonged bleeding. It should always be thought whenever only APTT is abnormal in screening tests. The treatment is usually available in almost all blood banks in form of fresh plasma.

\section{References}

1. Rosenthal RL, Dreskin OH, Rosenthal N. New haemophilia-like disease caused by deficiency of a third plasma thromboplastin factor. Proc Soc Exp Bio Med.1953;82(1):171-174.

2. Seligsohn U. High gene frequency of factor XI (PTA) deficiency in Ashkenazi Jews. Blood. 1978;51(6):1223-1228.

3. Seligsohn $U$, Modan $M$. Definition of the population at risk of bleeding due to factor $\mathrm{XI}$ deficiency in Ashkenazi Jews and the value of activated partial thromboplastin time in its detection. Isr J Med Sci. $1981 ; 17(6): 413$.

4. Rodgers GM, Grenberg CS. Inherited 
coagulation disorders. In: Greer JP, Foerster J, John N, Lukens JN (eds). Wintrobe's clinical hematology. $10^{\text {th }}$ ed. New York: LippincottRaven; 1998;1712.

5. Bolton-Maggs PH, Patterson DA, Wensley RT, Tuddenham EG. Definition of the bleeding tendency in factor $\mathrm{XI}$-deficient kindreds-a clinical and laboratory study. Thromb Haemost. 1995;73:194-202.
6. Bolton-Maggs $\mathrm{PH}$, Young Wan-Yin B, McCraw $A H$, Slack J, Kernoff PB. Inheritance and bleeding in factor XI deficiency. Br J Haematol. 1988;69:521-528.

7. Collins PW, Goldman E, Lilley P, Pasi KJ, Lee CA. Clinical experience of factor XI deficiency: the role of fresh frozen plasma and factor XI concentrate. Haemophilia. 1995; 1:227-231. 